\title{
Improvement in Transfer Performance for Antireflection Structured Films with Antifouling Properties via Partial-filling Technique
}

\author{
Hikari Eto $^{1}$, Shin Hiwasa ${ }^{2}$, and Jun Taniguchi ${ }^{*}$ \\ ${ }^{1}$ Department of Applied Electronics, Tokyo University of Science, \\ 6-3-1 Niijuku, Katsushika-ku, Tokyo 125-8585, Japan \\ ${ }^{2}$ Autex Co., Ltd., 16-5 Tomihisa-chou, Shinjuku-ku, Tokyo 162-0067, Japan \\ *junt@te.noda.tus.ac.jp
}

\begin{abstract}
Ultraviolet nanoimprint lithography (UV-NIL) is the most effective technique for mass fabrication of antireflection structure (ARS) films. For ARS films applied on touch-panel devices such as mobile phones and tablet computers, however, it is necessary to protect them from fingerprints and dust. Therefore, a UV-curable epoxy resin has been developed with antifouling properties for use with UV-NIL. However, because the developed epoxy resin possesses a high adhesive strength that ultimately reduces the lifetime of nanopatterned molds such as those for ARS, the ARS films herein were fabricated by a partial filling technique. The technique of partial filling uses an incomplete filling ratio of resin, and the work herein specifies the lower and higher filling pressures used for the repetitive transfer test. As a result, up to 200 ARS film imprints were successfully fabricated that exhibited excellent performance using lower pressure filling UV-NIL; while the reflectance obtained was less than $0.5 \%$. In addition, the fabricated ARS films were evaluated for their antifouling properties and their transfer durability performance using the wipe test and the repeated transfer test, respectively.
\end{abstract}

Keywords: Antireflection structures, Nanoimprint lithography, Antifouling property

\section{Introduction}

Films made using antireflection structures (ARSs) are used to suppress surface reflections from flat panel displays, mobile phones, and tablet computers. One commonly-used ARS is the motheye structure comprising a nano-array of needles, which exhibits a high antireflection performance and will be used herein. To use these ARS films on mobile phones and tablet computers that are also touch-panel devices, however, it is necessary to protect the films from fingerprints and dust. Therefore, it is critical to use an ARS film that has an antifouling property, but producing such a film is difficult because most films used on flat panel display devices do not produce an antifouling effect. In addition, nanoscale ARSs tend to get buried under the antifouling coating. Furthermore, a sufficient hardness is required in an ARS to protect the touch-panel devices from contact damage.

To solve these problems, an ultraviolet (UV)curable epoxy resin has been developed with antifouling properties [1] for use with UV-NIL [2,3]. The developed resin possesses the required antifouling properties and contact toughness (pencil hardness 5-6 $\mathrm{H}$ ), but it also possesses a high adhesive strength that causes a reduced lifetime for the nanopatterned molds (e.g., the ARS molds). Therefore, a technique is required to increase the lifetime of the ARS mold. In previous studies [4-6], partial filling has been found an effective technique for lengthening the lifetime of the ARS mold. The term "partial filling" describes the incomplete filling ratio of resin used in the mold. Using "complete filling" results in a strong release force owing to the large surface area of the adhesion resin, which consequently shortens the mold lifetime. 
Conversely, partial filling makes it easy to release the film from the ARS mold because of the small surface area of the adhesion resin, which consequently increases the mold lifetime.

The present study investigated the correlation between the resin filling behavior and the durability of the ARS mold at various filling pressures in repetitive transfer tests. Further, antifouling properties were tested by wiping colored artificial fingerprint liquid off of the film.

\section{Experimental apparatus and method}

The ARS master mold was fabricated by irradiating an oxygen ion beam on a mirror-surface glassy carbon (GC; Tokai Carbon Co., Ltd.) substrate using an electron cyclotron resonancetype ion shower device (EIG-210ER, Ericonix Inc.) $[7,8]$. The processing conditions used for the ARS GC mold are listed in Table 1. The GC mold, fabricated at a high acceleration voltage $(700 \mathrm{~V})$, formed wide pitches that facilitated easy filling of the resin during UV-NIL.

Table 1. Processing conditions of ARS GC mold

\begin{tabular}{cc}
\hline \multicolumn{2}{c}{ Processing conditions } \\
\hline Used substrate & Glassy carbon \\
Gas & $\mathrm{O}_{2}$ \\
Acceleration voltage & $700 \mathrm{~V}$ \\
Machining time & $60 \mathrm{~min}$ \\
\hline
\end{tabular}

For the release treatment, a $30 \mathrm{~nm}$-thick chromium layer was vacuum deposited as a metal layer on the surface of the processed GC by a vacuum evaporation machine (VPC-260F; ULVAC KIKO, Inc.) [9-12]. Thereafter, the mold was held in the liquid phase at $1 \mathrm{wt} \%$ Optool DSX (Daikin Industries, Ltd.) for $1 \mathrm{~h}$, the vapor phase for $12 \mathrm{~h}$, was baked at $120{ }^{\circ} \mathrm{C}$ for $5 \mathrm{~min}$, and was finally immersed in a fluorinated solvent NOVEC 7300 (3M Japan Ltd.) [13]. Figure 1 schematically shows the release treatment of the ARS GC mold.

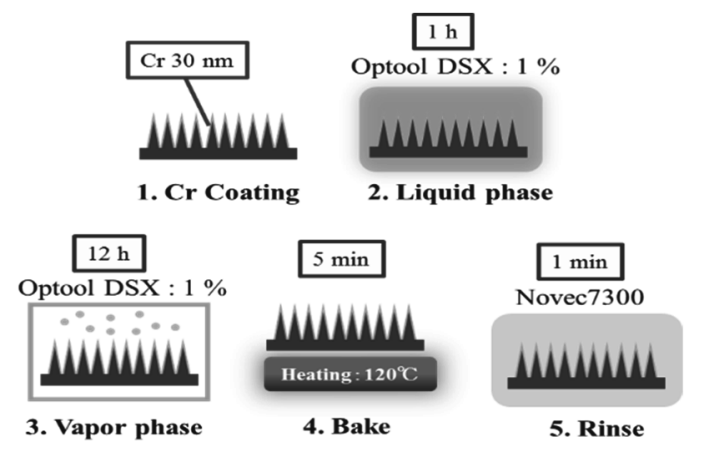

Fig. 1. Release treatment of ARS GC mold.
The ARS films were fabricated using a UVcurable resin with antifouling properties. The resin has been previously referred to as JST-FD-52-4K33 , and will be referred to herein as $52-4 \mathrm{~K}-33$. Resin $52-4 \mathrm{~K}-33$ consists of a blend of a cationicallypolymerizable UV-curable resin and an epoxymodified fluorinated resin, where the former is an alicyclic epoxy resin. Several alicyclic epoxy resins were blended for this study, and one typical alicyclic epoxy resin is 3,4-epoxycyclohexylmethyl-3,4epoxycyclohexanecarboxylate. The fluorinated component of 52-4K-33 segregates at the surface of the cured resin after heat treatment and imparts an antifouling property to the surface of the resin $[14,15]$.

First, the height of the ARS was studied for the varying imprint pressures of 0.03 ("low"), 0.06 , $0.17,0.28,0.56$, and 0.84 ("high") MPa. To meet the appropriate UV-NIL conditions, we employed a filling time of $15 \mathrm{~s}$, a UV dose of $4800 \mathrm{~mJ} / \mathrm{cm}^{2}$, a UV irradiation time of $5 \mathrm{~s}$, and a UV-curable resin of the 52-4K-33 type (viscosity of 60-75 $\mathrm{mPa} \cdot \mathrm{s}$ and pencil hardness of 5-6 H). The next step was to select an adequate filling pressure to further improve the life expectancy of the ARS mold.

The UV-NIL process was performed repeatedly using the ARS GC mold to examine its rate of deterioration. A parallel-plate-type UV-NIL machine (Mitsui Electric Co., Ltd.) was used to repeatedly imprint the ARS onto a polyester film (thickness $=100 \mu \mathrm{m}$; Cosmoshine A4300; Toyobo Co., Ltd.). However, the substrate with a film this thick tends to exhibit a low transparency and high reflectivity. Therefore, the first and the 200th imprints at the "low" filling pressure (0.03 MPa), and the first and 5th imprints at the "high" filling pressure $(0.84 \mathrm{MPa})$ were transferred to the polyester with the thin film (thickness $=50 \mu \mathrm{m}$ ) to measure the reflectance. Figure 2 shows the repeated UV-NIL process.

The low (0.03 MPa) and high (0.84 MPa) filling pressures of resin were studied to investigate their effect on the ARS mold lifetime. First, a layer of 52$4 \mathrm{~K}-33$ resin was applied to the ARS GC mold and covered with a polyester film layer. The assembly was then pressed at a pressure of either 0.03 or 0.84 $\mathrm{MPa}$ in a parallel-plate-type UV-NIL machine and then exposed to UV radiation, whereupon the 52$4 \mathrm{~K}-33$ layer was finally released from the ARS GC mold. This process was repeated using the ARS GC mold until the 52-4K-33 resin began to adhere to the ARS GC mold surface because of its repeated use, at which point the process with the lower or higher 
filling pressures in the UV-NIL machine was stopped.

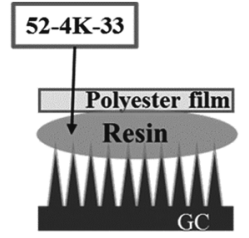

(a) Initial position (b) Pressed

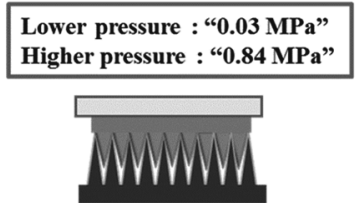

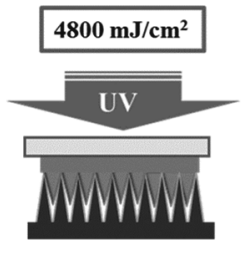

(c) Ultraviolet curing

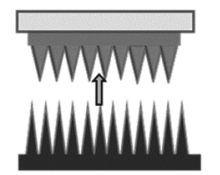

(d) Release

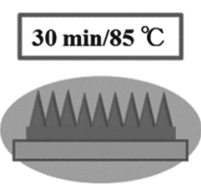

(e) Baking
Fig. 2. Repeated fabrication of ARS film.

To assess the ARS GC mold lifetime, the surface reflectance of the ARS films was measured using a UV-visible-infrared

(UV-Vis-IR) spectrophotometer in the spectral range of $300-1000$ $\mathrm{nm}$ at a $5^{\circ}$ angle of incidence (Solidspec-3700; Shimadzu Corp.), which was capable of highprecision measurements using an integrating sphere. Further imaging, including before-and-after images of the UV-NIL ARS GC mold pattern and the replicated ARS films filled at the low and the high pressure, were also obtained using a scanning electron microscope (SEM; ERA-8800FE, Elionix Co.).

The $52-4 \mathrm{~K}-33$ contains a fluorine polymer, and this fluorinated component segregates at the cured resin surface after heat treatment. The 52-4K-33 exhibits an antifouling effect at the surface of the resin, which can be generated after solidification with UV illumination. In addition, the liquid phase resin has no antifouling effect. To produce an antifouling effect at the resin surface, baking of the replicated ARS film was carried out at $85^{\circ} \mathrm{C}$ for 30 $\min [1,12]$.

Next, we fabricated the artificial fingerprint liquid used to create the stain for a wipe-off test. To make a visible stain, $0.1 \mathrm{wt} \%$ of Rhodamine B was added to give color to the artificial fingerprint liquid fabricated in accordance to the JIS K2246 standard. This fingerprint liquid was dropped on the ARS films and wiped off using Kimwipe ${ }^{\mathrm{TM}}$ S-200 (Nippon Paper Crecia Co., LTD.).

\section{Results and discussion}

Figure 3 shows the top view and the view when tilted by $75^{\circ}$ of the ARS GC mold used as the master mold. The ARS needle diameter, needle pitch, hole pitch and height of the master ARS mold were 50, 140,200 , and $1000 \mathrm{~nm}$, respectively. In addition, the needle pitch (i.e., inter-needle distance) of the ARS GC mold was $140 \mathrm{~nm}$, and the needle tips appear as white dots in Fig. 3 (top view), whereas the hole in which resin is filled appears as dark spaces in the same image. The hole pitch of the GC mold is 200 $\mathrm{nm}$, which coincides with the needle pitch of the ARS film transferred from the GC mold. The reflectance of the master mold was approximately $0.5 \%$ at visible-light wavelengths.

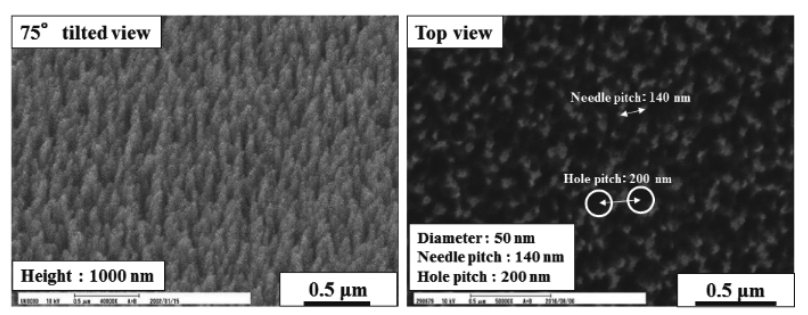

Fig. 3. SEM photos of the master mold.

Figure 4 shows the different ARS heights for varying imprint pressures. The height of the ARS tends to be greater when the imprint pressure increased, which changed the filling rate. The ARS height was approximately $450 \mathrm{~nm}$ for the low pressure of $0.03 \mathrm{MPa}$ and approximately $720 \mathrm{~nm}$ for the high pressure of $0.84 \mathrm{MPa}$. To evaluate of the length of the mold lifetime, the filling pressures of 0.03 and $0.84 \mathrm{MPa}$ were selected to create lower and higher pressure-filling UV-NIL, respectively. This signifies that changing the filling pressure can control the height of the ARS structure.

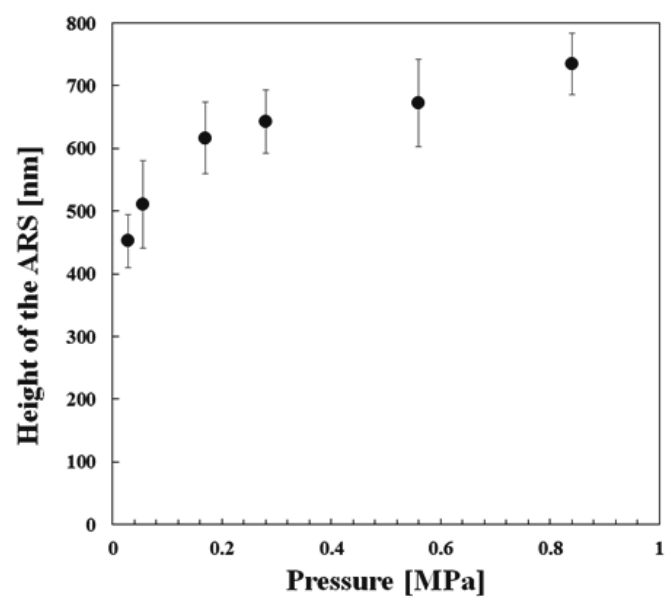

Fig. 4. ARS height in relation to pressure. 
Figures 5 and 6 show selected SEM photos of the low-pressure-filled ARS (1st (a) and 200th (b) imprints) and the high-pressure-filled ARS (1st (a) and 5 th (b) imprints) of $52-4 \mathrm{~K}-33$ resin. The $75^{\circ}$ tilted view of the SEM photos show that with the low-pressure filling it is possible to repeatedly transfer the imprint 200 times without any significant change in the ARS pattern. With the high-pressure filling, however, the imprint could be repeatedly transferred only 5 times. In addition, the tip of the needle is close owing to the greater needle height, which cause to widen the pitch, in the highpressure filling (Fig. 6). Therefore, the low-pressure filling was suitable for forming the ARS via UVNIL.

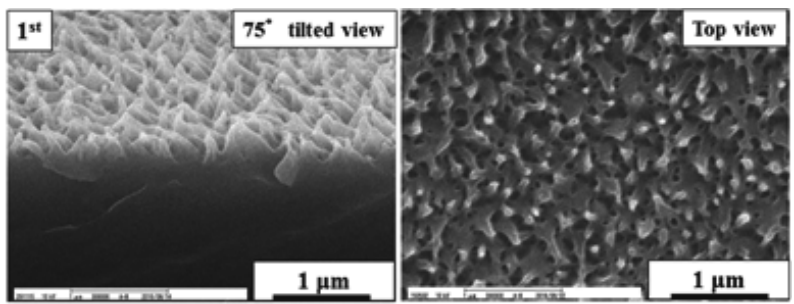

(a)

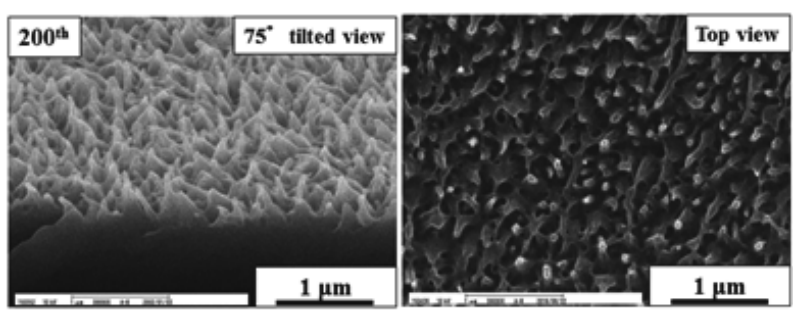

(b)

Fig. 5. SEM images of ARS films filled with $0.03 \mathrm{MPa}$ of pressure, showing the (a) 1st and (b) 200th imprint.

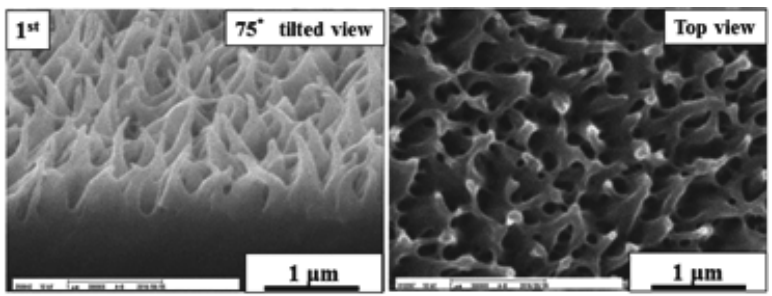

(a)

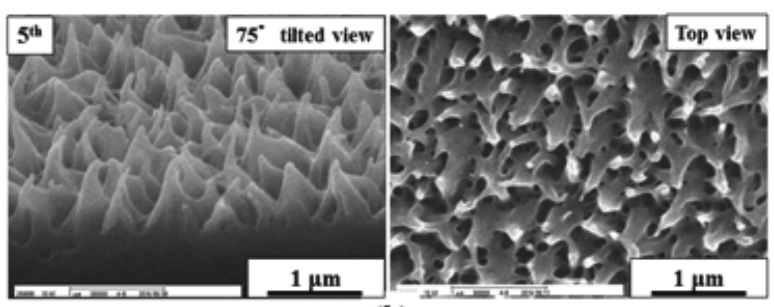

(b)

Fig. 6. SEM images of ARS films filled with $0.84 \mathrm{MPa}$ of pressure, showing the (a) 1st and (b) 5 th imprint.
Table 2 summarizes the values of height, diameter, and pitch for the ARS GC mold, and for the films produced by low- and high-pressure-filled ARS. The values show the feasibility of changing the structure height by varying the filling pressure. The value of the pitch corresponding to low- and highpressure filling was approximately $200 \mathrm{~nm}$, which coincides with the spaces of the GC master mold. Thus, it was confirmed that a highly accurate imprint was possible using UV-curable resin with antifouling properties.

Table 2. Morphologies of ARS GC mold and the low- and high-pressure-filled ARS films

\begin{tabular}{|c|c|c|c|}
\hline & $\begin{array}{l}\text { Height } \\
{[\mathrm{nm}]}\end{array}$ & $\begin{array}{c}\text { Diameter } \\
{[\mathrm{nm}]}\end{array}$ & $\begin{array}{l}\text { Pitch } \\
{[\mathrm{nm}]}\end{array}$ \\
\hline ARS GC mold & 1000 & 50 & 200 \\
\hline $\begin{array}{l}\text { Lower pressure } \\
\text { filled ARS_1 }\end{array}$ & 410 & 60 & 190 \\
\hline $\begin{array}{c}\text { Lower pressure } \\
\text { filled } \\
\text { ARS_200 }\end{array}$ & 420 & 70 & 210 \\
\hline $\begin{array}{c}\text { Higher } \\
\text { pressure filled } \\
\text { ARS_1 } 1^{\text {st }}\end{array}$ & 790 & 70 & 220 \\
\hline $\begin{array}{c}\text { Higher } \\
\text { pressure filled } \\
\text { ARS } 5^{\text {th }}\end{array}$ & 780 & 70 & 220 \\
\hline
\end{tabular}

The reflectivity values of the selected lowpressure-filled ARSs (1st and 200th imprints) and high-pressure-filled ARSs (1st and 5th imprints) are shown in Fig. 7. In this experiment, the ARS films were transferred to thin polyester films $(50 \mu \mathrm{m}$ thickness $)$, so the reflectance was low $(0.5 \%)$. The reflectance of the low-pressure-filled sample was not found to vary significantly between the first imprint cycle, the 200th imprint cycle, and the ARS GC mold. The reflectance of the high-pressurefilled sample was higher than that the lowpressure-filled sample for the first imprint cycle, however, and it tended to increase for every consecutive imprint with the highpressure filling method. As a result, the antireflection characteristics of the highpressure-filled ARS films are reduced owing to the wider the pitch because of the proximity to the tip of the needle (Fig. 6).

In addition, some resin remained on the ARS GC mold after the low-pressure filling cycles following the 225th imprint, and in the high-pressure filling cycles following the 5th imprint (Fig. 8). The 
adhesion of resin in the interstices of the ARS GC mold is visible in the photos taken after the imprinting process. These results show that a lower pressure during filling in UV-NIL extends the lifetime of the ARS GC mold compared to that of higher pressure during filling.

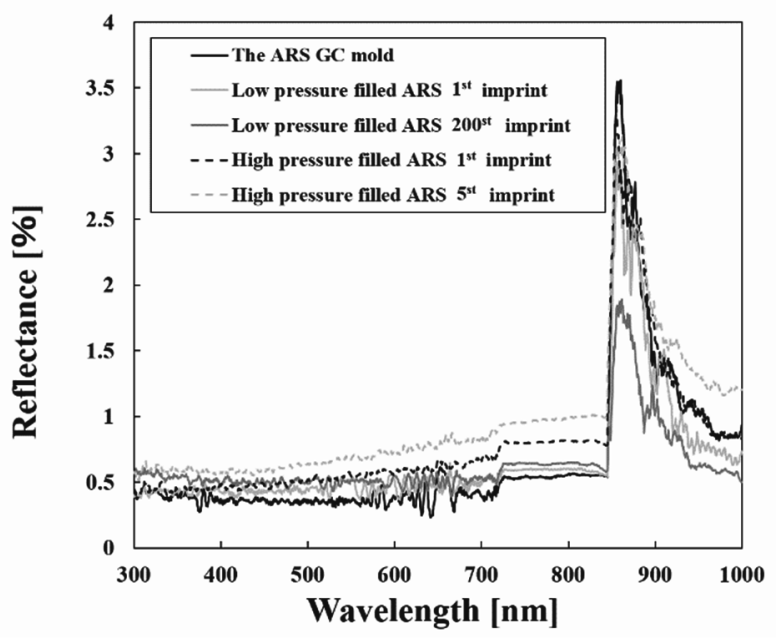

Fig. 7. Reflectivity of low- and high-pressure-filled ARS films and of the ARS GC mold.

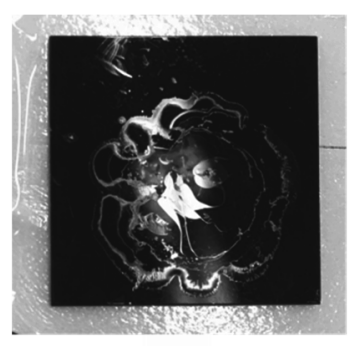

(a)

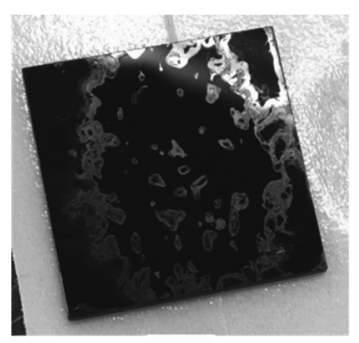

(b)
Fig. 8. Resin remaining on the ARS GC mold after the (a) low- and (b) high-pressure-filled cycle.

The verification of the antifouling property of the films are shown in Fig. 9, showing the colored artificial fingerprint liquid initially on the 100th transferred ARS surface with no baking treatment (Fig. 9(a)), and following a baking treatment (Fig. 9(c)). This colored artificial fingerprint liquid consists of an oil (e.g., a relatively high fatty acid), dust and 0.5 wt $\%$ of Rhodamine added as coloring; where the colored artificial fingerprint liquid area was subsequently wiped with a Kimwipe ${ }^{\mathrm{TM}}$ (Figs. 9(b) and 9(d)). In the first case with no baking, the colored artificial fingerprint liquid is not entirely wiped off (Fig. 9(b)). However, when the colored liquid was dropped on the baked ARS film surface the colored liquid was easily wiped away (Fig. 9 (d)). Therefore, the transferred 52-4K-33 surface acquires an antifouling property via baking.

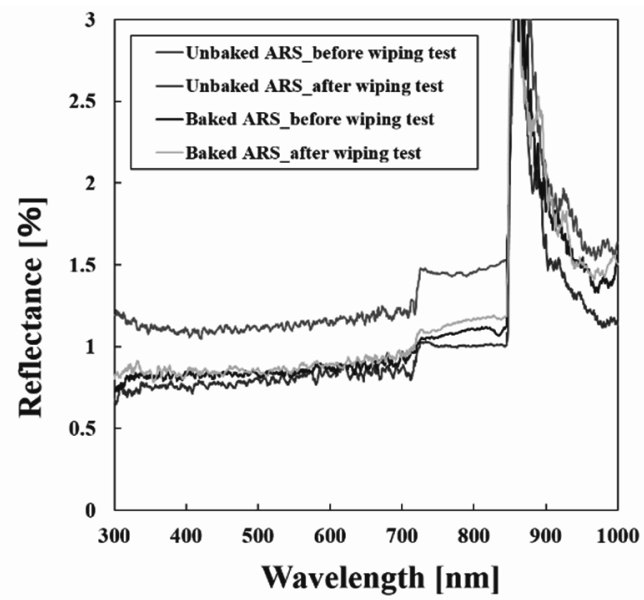

Fig. 9. For comparison, colored artificial fingerprint liquid dropped on the unbaked and baked ARS film. Unbaked film with (a) initial drop and (b) after wiping. Baked film with (c) initial drop and (d) after wiping.

The reflectance values were obtained again after the fingerprint wiping test on the unbaked and baked ARS films, and are shown in Fig. 10. In these measurements, the ARS films were transferred to the thick polyester films $(100 \mu \mathrm{m}$ thickness), so reflectance was higher than the results in Fig. 7. In the baked ARS films, the reflectance exhibits no significant change, whereas that in the unbaked film tends to be high because of the additional reflection caused by the residual colored artificial fingerprint liquid on the film surface.

Unbaked ARS film

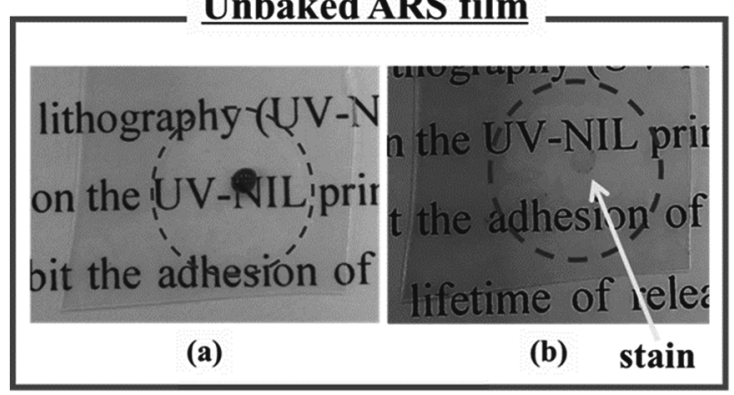

Baked ARS film

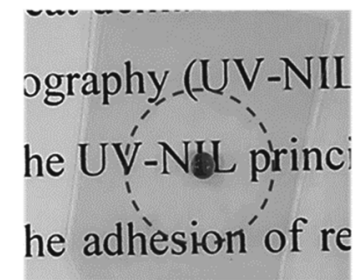

(c)

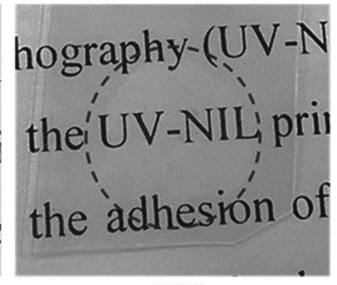

(d)
Fig. 10. Reflectivity after fingerprint wiping test on the unbaked and baked ARS films. 
Figures 11(a) and 11(b) show SEM images of the baked ARS film at a $75^{\circ}$ tilted view before and after the wiping tests, respectively. According to these SEM images, the ARS morphology does not change significantly following the wiping test. These images show that the fabricated ARS film is satisfactorily sturdy for wiping off marks because of its 5-6 $\mathrm{H}$ pencil hardness.

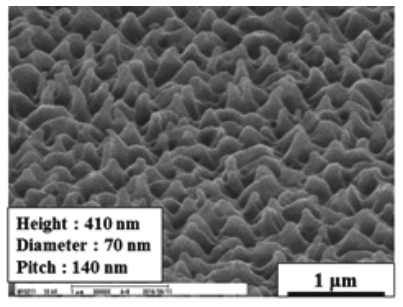

(a)

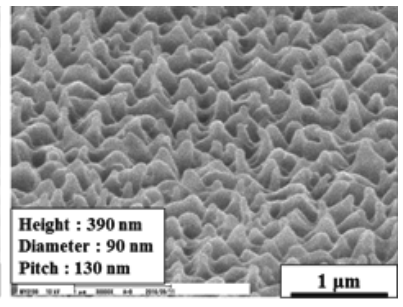

(b)
Fig. 11. SEM image of baked ARS film at a $75^{\circ}$ tilted view (a) before and (b) after the fingerprint wiping test.

\section{Conclusion}

In this study, transfer from an ARS GC mold with UV-NIL was successfully used to fabricate ARS films with antifouling properties. Increasing the imprint pressure to change the filling rate could control the height of the ARS. However, a higherpressure filling for the ARS was not suitable for repetitive imprint cycles because the high-pressurefilled resin for a high-aspect-ratio mold results in a strong release force owing to the large surface area of the adhesion resin, which consequently shortens its lifetime. In addition, the tip of the needle is close owing to the greater needle height, which cause to widen the pitch, in the high-pressure filling. Thus, the high-pressure-filled ARS films were caused high reflection. Therefore, the lower pressure-filled ARS films created using the partial filling technique have excellent performance, up to the 200th imprint; furthermore, the obtained reflectance was less than $0.5 \%$.

The antifouling effect of the ARS films allowed the artificial fingerprint liquid to be wiped off after a baking process. The ARS showed sufficient toughness, resisting breakage upon being rubbed.
The process outlined in this study is therefore suitable for fabricating ARS films that have antifouling properties and exhibit excellent performance.

\section{Acknowledgement}

This research was supported by the Adaptable and Seamless Technology Transfer Program through Target-driven R\&D, Japan Science and Technology Agency.

\section{References}

1. K. Yajima, K. Adachi, Y. Tsukahara, and J. Taniguchi, Microelectron. Eng., 110 (2013) 188.

2. S. Y. Chou, P. R. Krauss, W. Zhang, L. Guo, and L. Zhuang, J. Vac. Sci. Technol. B, 15 (1997) 2897.

3. H. Tan, A. Gilbertson, and S. Y. Chou, J. Vac. Sci. Technol. B, 16 (1998) 3926.

4. J. Takahashi and J. Taniguchi, Microelectron. Eng., 88 (2011) 2141.

5. N. B. A. T. Yusof and J. Taniguchi, Microelectron. Eng., 110 (2013) 163.

6. J. Taniguchi, Y. Kamiya, and N. Unno, J. Photopolym. Sci. Technol., 24 (2011) 104.

7. J. Taniguchi, Y. Nemoto, and Y. Sugiyama, $J$. Nanosci. Nanotechnol., 9 (2009) 445.

8. J. Taniguchi, E. Yamauchi, and Y. Nemoto, $J$. Phys. Conf. Ser., 106 (2008) 012011.

9. N. Unno, H. Tamura, and J. Taniguchi, Microelectron. Eng., 97 (2012) 72.

10. N. Unno and J. Taniguchi, Microelectron. Eng., 87 (2010) 1019.

11. J. Taniguchi and N. Unno, J. Photopolym. Sci. Technol., 28 (2015) 547.

12. J. Taniguchi and T. Tsuji, J. Photopolym. Sci. Technol., 29 (2016) 221.

13. J. Takahashi and J. Taniguchi, Microelectron. Eng., 88 (2011) 2141.

14. Y. Otsuka, S. Hiwasa, and J. Taniguchi, Microelectron. Eng., 123 (2014) 192.

15. J. Taniguchi, Y. Otsuka, S. Nose, N. Unno, and S. Hiwasa, J. Photopolym. Sci. Technol., 28 (2015) 563. 